\title{
A NEW GENERATION OF ITERATIVE TRANSFORM ALGORITHMS FOR PHASE CONTRAST TOMOGRAPHY
}

\author{
Heinz H. Bauschke, ${ }^{1}$ Patrick L. Combettes, ${ }^{2}$ and D. Russell Luke ${ }^{3}$ \\ ${ }^{1}$ Department of Mathematics and Statistics, University of Guelph, Guelph, Ontario N1G 2W1, Canada \\ ${ }^{2}$ Laboratoire Jacques-Louis Lions, Université Pierre et Marie Curie - Paris 6, 75005 Paris, France \\ ${ }^{3}$ University of Delaware Department of Mathematical Sciences Newark, DE 19716-2553, USA. *
}

\begin{abstract}
In recent years, improvements in electromagnetic sources, detectors, optical components, and computational imaging have made it possible to achieve three-dimensional atomicscale resolution using tomographic phase-contrast imaging techniques. These greater capabilities have placed a premium on improving the efficiency and stability of phase retrieval algorithms for recovering the missing phase information in diffraction observations. In some cases, so called $d i$ rect methods suffice, but for large macromolecules and nonperiodic structures one must rely on numerical techniques for reconstructing the missing phase. This is the principal motivation of our work. We report on recent progress in algorithms for iterative phase retrieval. The theory of convex optimisation is used to develop and to gain insight into counterparts for the nonconvex problem of phase retrieval. We propose a relaxation of averaged alternating reflectors and determine the fundamental mathematical properties of the related operator in the convex case. Numerical studies support our theoretical observations and demonstrate the effectiveness of the newer generation of algorithms compared to the current state of the art.
\end{abstract}

\section{INTRODUCTION}

The phase retrieval problem is an inverse problem, well known in optics, that has received renewed interest in applications to nonperiodic scatterers and macromolecules. In a typical $\mathrm{x}$-ray crystallography experiment, for example, a crystalline specimen is illuminated with a monochromatic $\mathrm{x}$ ray and the resulting diffraction pattern is recorded. In the far field of the crystal the complex amplitude of the diffracted $\mathrm{x}$ rays is equal to the (scaled) Fourier transform of the electron density distribution of the specimen. The problem is that only the intensity of the diffracted field can be measured. The missing phase information is critical for

\footnotetext{
*This author's work was supported in part by post doctoral fellowships from Universität Göttingen and PIMS. Send correspondence to: rluke@math. udel. edu
}

determining the electron density. In some cases, such as $\mathrm{x}$ ray crystallography of "small" (relative to the source wavelength) periodic molecules, it is possible to determine the electron density by what are referred to as direct methods. For large macromolecules and nonperiodic structures, however, one must rely on numerical techniques for reconstructing the missing phase. So called iterative transform methods are well established generic iterative techniques for recovering the phase in a variety of settings. Recent trends in phase contrast tomography have placed a premium on improving the efficiency and stability of these types of algorithms [1,2].

In this work we derive stable and fast new strategies for phase retrieval. Recently, we identified two important methods for phase retrieval, namely Fienup's Basic InputOutput (BIO) and Hybrid Input-Output (HIO) algorithms, with classical convex projection methods and suggested that further connections between convex optimization and phase retrieval should be explored [3]. Following up on this fundamental work, we have derived several new projection-based methods, termed the Hybrid Projection Reflection (HPR) algorithm [4], and the Relaxed Averaged Alternating Reflection (RAAR) algorithm [5]. These methods are particularly effective for solving phase retrieval problems featuring nonnegativity constraints in the object domain.

A precise statement of the leading algorithms is given in Section 2 In this same section we provide a terse outline of the mathematical justification for the HPR and RAAR algorithms. In Section 3 we demonstrate the effectiveness of the algorithms.

\section{PHASE RETRIEVAL AND ITERATIVE TRANSFORM ALGORITHMS}

\subsection{Phase retrieval}

In its general form, the signal recovery problem is to estimate the original form of a signal $u$ in a functional space $\mathcal{L}$ from the measurements of physically related signals and a priori information. In phase retrieval problems, the mea- 
surements consist of the modulus $m$ of the Fourier transform $\mathcal{F} u$ of $u$. In other words, the imaging model is described by the relationship

$$
|\mathcal{F} u|=m,
$$

and $u$ is commonly referred to as the object or input of the imaging model.

A general signal space that appropriately models the underlying physics is the complex Hilbert space $\mathcal{L}=$ $L^{2}\left[\mathbb{R}^{N}, \mathbb{C}\right]$. Hence, a signal $u$ in $\mathcal{L}$ is a square-integrable function mapping a continuous variable $x \in \mathbb{R}^{N}$ to a complex number $u(x) \in \mathbb{C}$. The set of signals that satisfy the Fourier domain constraint (1) is

$$
M=\{v \in \mathcal{L}:|\mathcal{F} v|=m \text { a.e. }\} .
$$

In addition to the imaging model, an important piece of information that is typically available in phase retrieval problems is that the support of $u$ is contained in some set $D \subset \mathbb{R}^{N}$. If we let $1_{E}$ denote the characteristic function of a set $E \subset \mathbb{R}^{N}$ and $\complement E$ its complement, this object domain constraint confines $u$ to the set

$$
S=\left\{v \in \mathcal{L}: v \cdot 1_{\mathrm{C}_{D}}=0\right\} .
$$

It is also sometimes useful to include the additional constraint that $u$ be nonnegative, as would be natural when $u$ represents the electron density of a crystal. Writing this in terms of constraints, we have $u_{*} \in S_{+} \subset \mathcal{L}$, where $S_{+}$is the set of nonnegative functions in $\mathcal{L}$ with support on $D$. The sets $S$ and $S_{+}$are referred to as the physical domain constraints.

\subsection{Feasibility problems and fixed point algorithms}

We formulate the problem of phase retrieval as a feasibility problem:

$$
\text { find } u \in S_{+} \cap M \text {. }
$$

This formulation exhibits the phase retrieval problem as a problem of finding a point in the intersection of constraint sets, i.e., a set theoretic estimation problem. In mathematics (especially in optimization) problems of this kind are called feasibility problems.

Iterative transform techniques are built upon combining projections onto the sets $S_{+}$and $M$ in some fashion. They can usually be put into the form $u_{n+1}=\mathcal{T} u_{n}$ where $\mathcal{T}$ is a generic operator in which the projections and averaging operations are embedded (see [3, 4, 6]). For added control and flexibility, one often includes a relaxation strategy parameterised by $\beta$. We write the relaxed operator with generic, single parameter relaxation strategy $\mathcal{V}$ (there can be infinitely many such strategies) as $\mathcal{V}(\mathcal{T}, \beta)$. In order effectively to exploit relaxations for improved algorithm performance it is necessary to understand the mathematical properties of the operator $\mathcal{V}(\mathcal{T}, \beta)$. We return to this issue at the end of this section.

\subsection{Projectors and Reflectors}

The operators we study are built upon projectors and reflectors. Denote by $P_{C}$ an arbitrary but fixed selection, or projector, from the possibly multi-valued projection onto a subset $C$ of $\mathcal{L}$. Closely related is the corresponding reflector with respect to $C$

$$
R_{C}=2 P_{C}-I
$$

where $I$ is the identity operator. By definition, for every $u \in \mathcal{L}, P_{C}(u)$ is the midpoint between $u$ and $R_{C}(u)$. Specialising to our application, the projector, $P_{M} u$, of a signal $u \in \mathcal{L}$ onto the Fourier magnitude constraint set $M$ is given by where

$$
P_{M}(u)=\mathcal{F}^{-1}\left(\widehat{v}_{0}\right)
$$

where

$$
\widehat{v}_{0}(\xi)= \begin{cases}m(\xi) \frac{\mathcal{F} u(\xi)}{|\mathcal{F} u(\xi)|}, & \text { if } \mathcal{F} u(\xi) \neq 0 ; \\ m(\xi), & \text { otherwise }\end{cases}
$$

Here, $\mathcal{F}^{-1}$ is the discrete inverse Fourier transform and $\widehat{v}_{0}$ a selection from the multi-valued Fourier domain projection. The projection of a signal $u \in \mathcal{L}$ onto $S_{+}$is single-valued (since $S_{+}$is convex), and is given by

$\left(\forall x \in \mathbb{Z}^{N}\right)\left(P_{S_{+}}(u)\right)(x)= \begin{cases}\max \{0, u(x)\}, & \text { if } x \in D ; \\ 0, & \text { otherwise }\end{cases}$

\subsection{Iterative Transform Algorithms}

One of the best known iterative transform algorithms is Fienup's Hybrid Input-Output algorithm (HIO) [7]. We use this as our benchmark for performance. In the present setting, HIO is given as: for all $\forall x \in \mathbb{Z}^{N}$

$$
u_{n+1}(x)=\left\{\begin{array}{r}
\left(P_{M}\left(u_{n}\right)\right)(x), \text { if } x \in D \\
\text { and }\left(P_{M}\left(u_{n}\right)\right)(x) \geq 0 ; \\
u_{n}(x)-\beta_{n}\left(P_{M}\left(u_{n}\right)\right)(x), \text { otherwise. }
\end{array}\right.
$$

There have been several attempts to identify the HIO algorithm with a broader class of relaxation strategies that can be written as fixed point iterations, that is, in the form $u_{n+1}=\mathcal{V}\left(\mathcal{T}, \beta_{n}\right) u_{n}$. We proved that, when only a support constraint, as opposed to support and nonnegativity, is applied in the physical domain, then the HIO algorithm with $\beta=1$ corresponds to the classical Douglas-Rachford algorithm for which convergence results in the convex setting are well known [3]. In a subsequent article [4] we proved that, for physical domain support constraints only, 
the HIO algorithm corresponds to a particular relaxation of the Douglas-Rachford algorithm, that is, for all $x \in \mathbb{Z}^{N}$

$$
u_{n+1}(x)= \begin{cases}\left(P_{M}\left(u_{n}\right)\right)(x), & \text { if } x \in D \\ u_{n}(x)-\beta_{n}\left(P_{M}\left(u_{n}\right)\right)(x), & \text { otherwise, }\end{cases}
$$

is equivalent to

$u_{n+1}=\frac{1}{2}\left(R_{S}\left(R_{M}+\left(\beta_{n}-1\right) P_{M}\right)+I+\left(1-\beta_{n}\right) P_{M}\right)\left(u_{n}\right)$.

A subtlety of these algorithms (one that is often confused in the optics literature) is that seemingly simple changes in the constraint structure of the problem leads to very different algorithms when written in the form (9) and (10). Indeed, replacing $S$ in 111 with $S_{+}$yields

$u_{n+1}=\frac{1}{2}\left(R_{S_{+}}\left(R_{M}+\left(\beta_{n}-1\right) P_{M}\right)+I+\left(1-\beta_{n}\right) P_{M}\right)\left(u_{n}\right)$.

It has been incorrectly assumed that recurrence $(12)$ is equivalent to (9]. Instead, we have shown in [4, Proposition 2] that (12) is an entirely new algorithm, what we call the Hybrid Projection Reflection (HPR) algorithm, for all $x \in \mathbb{Z}^{N}$

$u_{n+1}(x)=\left\{\begin{array}{c}\left(P_{M}\left(u_{n}\right)\right)(x), \text { if } x \in D \text { and } \\ \quad\left(R_{M}\left(u_{n}\right)\right)(x) \geq\left(1-\beta_{n}\right)\left(P_{M}\left(u_{n}\right)\right)(x) ; \\ u_{n}(x)-\beta_{n}\left(P_{M}\left(u_{n}\right)\right)(x), \quad \text { otherwise. }\end{array}\right.$

It is easy to see by comparing (13) with (9) that these are fundamentally different algorithms. Moreover, a reformulation of (9) in terms of a fixed point iteration does not appear to be possible due to the nonlinearity of the $P_{S_{+}}$operator [4].

We show below that HPR is a promising alternative to $\mathrm{HIO}$, however we have not found an analysis that suggests how to choose the relaxation parameter $\beta$. Moreover, at the initial phase of the iteration, the HIO algorithm appears to reach a neighbourhood of a solution to the feasibility problem (4) in fewer iterations than HPR, though at later stages of the iteration HPR clearly delivers better quality images more stably.

To address these drawbacks to the HPR algorithm, we focus on the intermediate stage of these algorithms where HIO appears to outperform HPR. The RAAR algorithm discussed next achieves improved performance at the intermediate stage and the superior stability/quality of the HPR algorithm at later iterations through an analytically motivated relaxation strategy. The relaxed averaged alternating reflection (RAAR) algorithm is a relaxation of the averaged alternating reflection algorithm studies in [6] and is given by the following: given any $u_{0} \in \mathcal{L}$, generate the sequence $u_{0}, u_{1}, u_{2}, \ldots$ by

$$
u_{n+1}=V\left(\mathcal{T}_{*}, \beta_{n}\right) u_{n}
$$

where

$$
V\left(\mathcal{T}_{*}, \beta\right)=\beta \mathcal{T}_{*}+(1-\beta) P_{M} \quad \text { and } \quad \mathcal{T}_{*}=\frac{1}{2}\left(R_{S_{+}} R_{M}+I\right)
$$

For $\beta=1$ the RAAR and HPR algorithms are equivalent. For $\beta \neq 1$ the RAAR algorithm is fundamentally different than HPR. In [5, Proposition 2.1] Luke shows that the recursion (14) is equivalent to the following algorithm: for all $x \in \mathbb{Z}^{N}$

$$
u_{n+1}(x)=\left\{\begin{aligned}
&\left(P_{M}\left(u_{n}\right)\right)(x), \text { if } x \in D \\
& \text { and }\left(R_{M}\left(u_{n}\right)\right)(x) \geq 0 \\
& \beta_{n} u_{n}(x)-\left(1-2 \beta_{n}\right)\left(P_{M}\left(u_{n}\right)\right)(x), \text { else. }
\end{aligned}\right.
$$

\section{COMPARISONS}

The principal criteria we use to assess the performance of these algorithms are iteration counts, quality of solutions and stability. Preliminary numerical results indicate that, once in the neighbourhood of a solution, the HPR iterates stay in that neighbourhood [4], unlike iterates of HIO. Moreover, by both the error metric and the subjective eyeball norm, eventually the images delivered by the HPR algorithm are superior to those of the HIO algorithm [4]. The relaxed RAAR algorithm is superior to HIO and HPR with respect to stability, though image quality suffers with greater relaxation.

Our goal with the RAAR algorithm is to use dynamic relaxations to shorten the initial "warm-up" phase of the HPR algorithm and to stabilise the algorithm near a local solution. The relaxation strategy we consider is

$$
\beta_{n+1}=\beta_{0}+\left(1-\beta_{0}\right)\left(1-\exp \left(-(n / 7)^{3}\right)\right) .
$$

The data consists of the support/nonnegativity constraint, shown in Figure 3(c), and Fourier magnitude data $m$, shown in Figure 3 (b), with additive noise $\eta$ - a symmetric, randomly generated array with a zero mean Gaussian distribution. Following the experimental design of [4], the signal-to-noise ratio (SNR) is $20 \log _{10}\|m\| /\|u\|=34 \mathrm{~dB}$. We compute the mean value of the error measure $E_{S+}$ over 100 trials with different realizations of the noise and the same initial guess.

We compare the mean behaviour over 100 iterations of two sets of realizations of the algorithms, each corresponding to different relaxation strategies, $\beta=0.75, \beta=$ $0.87, \beta=0.99$ and variable $\beta_{n}$ governed by 17 with $\beta_{0}=0.75$. The average value of the error metric at iteration $n$,

$$
E_{S_{+}}\left(x_{n}\right)=\frac{\left\|P_{S_{+}}\left(P_{M}\left(u_{n}\right)\right)-P_{M}\left(u_{n}\right)\right\|^{2}}{\left\|P_{M}\left(u_{n}\right)\right\|^{2}}
$$

is shown in Figure 1. 
(a)

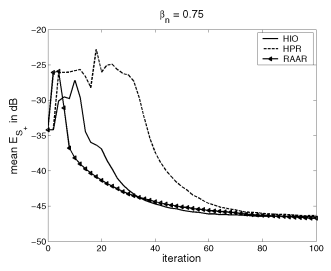

(c)

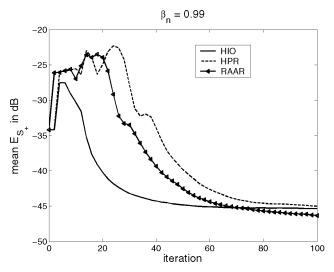

(b)

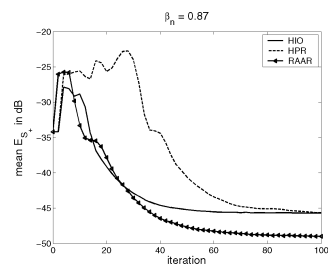

(d)

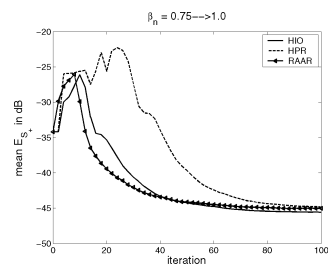

(a)

$$
\text { HIO }
$$

HPR

RAAR
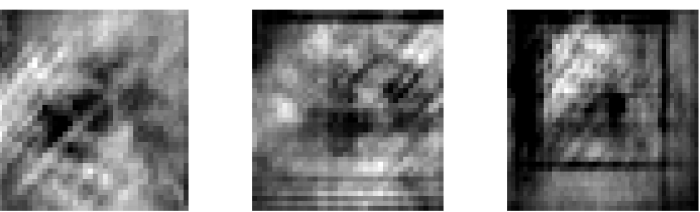

(b)
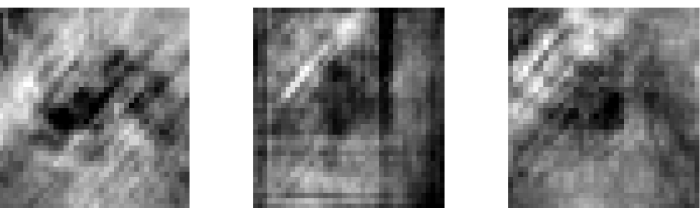

Fig. 1. Error metric $E_{S_{+}}\left(x_{n}\right)$ averaged over 100 realizations of noise $(\mathrm{SNR}=34 \mathrm{~dB})$. For (a)-(c) the relaxation parameter for the respective algorithms, $\beta_{n}$, is fixed. For (d) $\beta_{n}$ varies from 0.75 to 1.0 according to 17 ).

\section{REFERENCES}

[1] P. Jonas and A. K. Louis, "Phase contrast tomography using holographic measurements," Inv. Prob., vol. 20, pp. $75-102,2004$.

[2] A. W. Stevenson, T. E. Gureyev, D. Paganin, S. W. Wilkins, T. Weitkamp, A. Snigirev, C. Rau, I. Snigireva, H. S. Youn, I. P. Dolbnya, W. Yun, B. Lai, R. F. Garrett, D. J. Cookson, K. Hyodo, and M. Ando, "Phasecontrast X-ray imaging with synchrotron radiation for materials science applications," Nucl. Instrum. \& Meth. Phys. Res. B, vol. 199, pp. 427-435, 2003.

[3] H. H. Bauschke, P. L. Combettes, and D. R. Luke, "Phase retrieval, error reduction algorithm and Fienup variants: a view from convex feasibility," J. Opt. Soc. Am. A., vol. 19, no. 7, pp. 1334-45, 2002.

[4] H. H. Bauschke, P. L. Combettes, and D. R. Luke, "A hybrid projection reflection method for phase retrieval," J. Opt. Soc. Am. A., vol. 20, no. 6, pp. 1025-34, 2003.

[5] D. R. Luke, "Relaxed averaged alternating reflections for diffraction imaging," Inv. Prob., to appear.

[6] H. H. Bauschke, P. L. Combettes, and D. R. Luke, "Finding best approximation pairs relative to two closed convex sets in hilbert spaces," J. Approx. Theory, vol. 127, pp. 178-92, 2004.

[7] J. R. Fienup, "Phase retrieval algorithms: a comparison," Appl.Opt., vol. 21, no. 15, pp. 2758-2769, 1982.

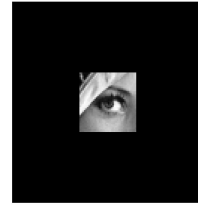

(a)

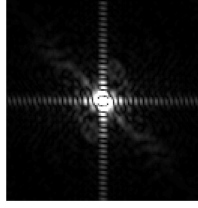

(b)

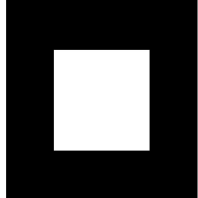

(c)
Fig. 3. Original images and corresponding data used for the comparison of the HIO and HPR algorithms. (a) True $38 \times 38$ pixel signal, zero-padded to $128 \times 128$. (b) the noiseless Fourier magnitude data $m$ corresponding to (a). (c) object domain support constraint (and initial guess), $64 \times 64$ pixels. 